The Astrophysical Journal Supplement Series, 158:80-92, 2005 May

(C) 2005. The American Astronomical Society. All rights reserved. Printed in U.S.A.

\title{
ELECTRON-ION RECOMBINATION RATE COEFFICIENTS AND PHOTOIONIZATION CROSS SECTIONS FOR ASTROPHYSICALLY ABUNDANT ELEMENTS. IX. Ni XXVI AND Ni XXVII FOR ULTRAVIOLET AND X-RAY MODELING
}

\author{
Sultana N. Nahar \\ Department of Astronomy, The Ohio State University, Columbus, OH 43210; nahar@astronomy.ohio-state.edu \\ Received 2004 November 21; accepted 2005 January 6
}

\begin{abstract}
The inverse processes of photoionization and electron-ion recombination of $(h \nu+\mathrm{Ni} \mathrm{xXVI} \leftrightarrow \mathrm{Ni} \mathrm{XXVII}+e)$ and $(h \nu+\mathrm{Ni}$ XXVII $\leftrightarrow \mathrm{Ni}$ XXVIII $+e)$ are studied using the unified method for the total recombination. The method subsumes both the radiative and dielectronic recombination processes and enables self-consistent sets of results for photoionization and electron-ion recombination by using the same wavefunction for these inverse processes. Photoionization cross sections $\left(\sigma_{\mathrm{PI}}\right)$, recombination cross sections $\left(\sigma_{\mathrm{RC}}\right)$, recombination collision strengths $\left(\Omega_{\mathrm{RC}}\right)$, and recombination rate coefficients $\left(\alpha_{\mathrm{RC}}\right)$ are obtained for ionization balance and spectral analysis of UV and X-ray lines. Level-specific photoionization cross sections and recombination rates are presented to enable accurate computation of recombination-cascade matrices for all fine-structure levels $n(\ell S L J)$ up to $n \leq 10: 98$ bound finestructure levels of $\mathrm{Ni}$ XXVI with $0 \leq l \leq 9,0 \leq L \leq 11$, and $1 / 2 \leq J \leq 17 / 2$, and 198 levels of Ni XXVII with $0 \leq l \leq 9,0 \leq L \leq 14$, and $0 \leq J \leq 10$. Total $\alpha_{\mathrm{RC}}$-values for Ni XXVI and Ni XXVII are compared with the existing values and are found to be in very good agreement. Total recombination rate coefficients for the hydrogen-like recombined ion, Ni XxVIII, are also presented. The calculations are carried out in the relativistic Breit-Pauli $\boldsymbol{R}$-matrix (BPRM) approximation with inclusion of radiation damping of resonances. With consideration of all details of the processes, the results, which include the level-specific $\sigma_{\mathrm{PI}}$ and $\alpha_{\mathrm{RC}}$ calculated for the first time, should be the most accurate for these ions.
\end{abstract}

Subject headings: atomic data — atomic processes — line: formation — X-rays: general

\section{INTRODUCTION}

From a study of photoionization and electron-ion recombination for Li- and He-like ions, similar to the earlier ones for C IV and C v (Nahar et al. 2000), O vi and O vII (Nahar \& Pradhan 2003), and Fe Xxv and Fe Xxv (Nahar et al. 2001), the results of cross sections and rates are presented for Ni XXVI and Ni XXVII. These inverse atomic processes of Li-like and He-like ions are of particular interest in X-ray astronomy for analysis of new observations by space-based observatories such as Chandra and $X M M-N e w t o n$, at photon energies and temperatures prevalent in high-temperature sources such as active galactic nuclei, supernova remnants, hot stellar coronae, etc. (e.g., Canizares et al. 2000).

The Li-like and He-like ions show distinctive features, compared with those of higher multielectron ions, in both photoionization and electron-ion recombination: (i) a featureless smooth structure is displayed until at high energies when narrow and dense resonances appear in separated $n$-complexes, and (ii) the low- $n$ resonances are radiationally damped because of higher radiative decay rates. It is important to consider relativistic fine-structure effects explicitly in the theoretical formulation to resolve the narrow resonances and include radiation damping of resonances. Photoionization cross sections, electronion recombination cross sections, and rate coefficients are obtained in the unified method for the total electron-ion recombination, accounting both radiative and dielectronic recombination ( RR and DR) processes, in the frame of the relativistic Breit-Pauli $\boldsymbol{R}$-matrix (BPRM) method (Nahar \& Pradhan 1992, 1994; Nahar 1996; Zhang et al. 1999). The unified method provides a single set of recombination rate coefficients taking into account both RR and DR in an ab initio manner for the entire temperature range of applications. The aim of the present series of reports (e.g., Nahar \& Pradhan 1997) is to study and present accurate atomic parameters for photoionization and total ( $e+$ ion) recombination for astrophysical models in a variety of applications.

\section{THEORY}

The unified method of electron-ion recombination (Nahar \& Pradhan 1992, 1994) yields self-consistent sets of atomic parameters for the inverse processes of photoionization and recombination for atoms and ions. Photorecombination of an incident electron with the target ion may occur through (i) nonresonant, background continuum, or radiative recombination (RR),

$$
e+\mathrm{X}^{++} \leftrightarrow h \nu+\mathrm{X}^{+}
$$

which is the inverse process of direct photoionization, or (ii) through a two-step recombination process via autoionizing resonances, i.e., dielectronic recombination (DR),

$$
e+\mathrm{X}^{++} \leftrightarrow\left(\mathrm{X}^{+}\right)^{* *} \leftrightarrow\left\{\begin{array}{c}
\text { (i) } e+\mathrm{X}^{++} \\
\text {(ii) } h \nu+\mathrm{X}^{+}
\end{array}\right\}
$$

The quasi-bound doubly excited autoionizing state, $\left(\mathrm{X}^{+}\right)^{* *}$, leads either to (i) autoionization, a radiationless transition to a lower target state with the electron going into a continuum, or (ii) radiative stabilization to a recombined bound state via radiative decay of the ion core, usually to the ground state, with the capture of the electron.

The unified method subsumes both the RR and DR processes. It considers photoionization from and recombination into the infinity of levels of the $(e+$ ion $)$ system. These 
recombined levels are divided into two groups: group A, bound levels with $n \leq n_{0}$ and all possible fine-structure $J \pi$ symmetries; and group B, levels with $n_{0}<n \leq \infty$, where $n_{0}$ is typically 10 .

Photoionization and recombination calculations are carried out in detail for all group A levels in the close-coupling (CC) approximation (e.g., Seaton 1987). In the CC approximation the target ion (core) is represented by an $N$-electron system. The total wavefunction, $\Psi(E)$, of the $(N+1)$ electron-ion system of symmetry $J \pi$ is represented in terms of an expansion of target eigenfunctions as

$$
\Psi(\text { ion }+e ; E)=A \sum_{i} \chi_{i}(\text { ion }) \theta_{i}+\sum_{j} c_{j} \Phi_{j}(\text { ion }+e),
$$

where $\chi_{i}$ is the target wavefunction for a specific level $J_{i} \pi_{i}$ and $\theta_{i}$ is the wavefunction for the $(N+1)$-th electron in a channel labeled as $S_{i} L_{i}\left(J_{i}\right) \pi_{i} k_{i}^{2} \ell_{i}(J \pi), k_{i}^{2}$ being its incident kinetic energy. The $\Phi_{j}$-values are the correlation functions of the $(N+1)$ electron system that account for short-range correlation and the orthogonality between the continuum and the bound orbitals.

In relativistic BPRM calculations, developed under the Iron Project (IP; Hummer et al. 1993), the set of $S L \pi$-values are recoupled for $J \pi$ levels of the (e+ion) system, followed by diagonalization of the Hamiltonian, $H_{N+1}^{\mathrm{BP}} \Psi=E \Psi$, where the BP Hamiltonian is

$$
H_{N+1}^{\mathrm{BP}}=H_{N+1}^{\mathrm{NR}}+H_{N+1}^{\mathrm{mass}}+H_{N+1}^{\mathrm{Dar}}+H_{N+1}^{\mathrm{so}} .
$$

The first term, $H_{N+1}^{\mathrm{NR}}$, is the nonrelativistic Hamiltonian,

$$
H_{N+1}^{\mathrm{NR}}=\sum_{i=1}^{N+1}\left\{-\nabla_{i}^{2}-\frac{2 Z}{r_{i}}+\sum_{j>i}^{N+1} \frac{2}{r_{i j}}\right\}
$$

and the additional one-body terms are

$$
\begin{aligned}
& \text { the mass correction term, } H^{\text {mass }}=-\frac{\alpha^{2}}{4} \sum_{i} p_{i}^{4}, \\
& \text { the Darwin term, } H^{\text {Dar }}=\frac{Z \alpha^{2}}{4} \sum_{i} \nabla^{2}\left(\frac{1}{r_{i}}\right),
\end{aligned}
$$

the spin-orbit interaction term, $H^{\mathrm{so}}=Z \alpha^{2} \sum_{i} \frac{1}{r_{i}^{3}} \boldsymbol{l}_{\boldsymbol{i}} \cdot \boldsymbol{s}_{\boldsymbol{i}}$,

respectively. The spin-orbit term splits the $L S$ term into finestructure components.

The positive and negative energy states (eq. [4]) define continuum or bound $\left(e+\right.$ ion) states: $E=k^{2}>0$ for continuum (scattering) channels and $E<0$ for bound states. The reduced matrix element for the bound-free transition,

$$
\left\langle\Psi_{B}|| \boldsymbol{D} \| \Psi_{F}\right\rangle
$$

can be obtained from the continuum wavefunction $\left(\Psi_{F}\right)$ and the bound wavefunction $\left(\Psi_{B}\right)$. Here $\boldsymbol{D}$ is the dipole operator, $\boldsymbol{D}_{L}=\sum_{i} \boldsymbol{r}_{\boldsymbol{i}}$, in length form, the sum is on the number of electrons. The photoionization cross section is obtained as

$$
\sigma_{\mathrm{PI}}=\frac{1}{g_{i}} \frac{4 \pi^{2}}{3 c} \omega \boldsymbol{S}
$$

where $g_{i}$ is the statistical weight factor of the initial bound state and $\boldsymbol{S}=\left|\left\langle\Psi_{B}|| \boldsymbol{D} \| \Psi_{F}\right\rangle\right|^{2}$ is the dipole line strength, For highly charged $\mathrm{H}$ - and the He-like recombining ions, the probability of radiative decay of an autoionizing state is often comparable to that of autoionization (typically $10^{12}-10^{14} \mathrm{~s}^{-1}$, as discussed in Nahar et al. 2000). With strong dipole allowed $2 p \rightarrow 1 s$ and $1 s 2 p\left({ }^{1} P_{1}^{o}\right) \rightarrow 1 s^{2}\left({ }^{1} S_{0}\right)$ transitions (e.g., Table 2 ) autoionizing resonances are radiatively damped to a significant extent. The radiative damping effects of all near-threshold resonances, up to effective quantum number $\nu \leq 10$, are considered using a resonance-fitting procedure (Sakimoto et al. 1990; Pradhan \& Zhang 1997; Zhang et al. 1999).

The photorecombination cross section, $\sigma_{\mathrm{RC}}$, is related to the photoionization cross section, $\sigma_{\mathrm{PI}}$, through the principle of detailed balance (the Milne relation) as

$$
\sigma_{\mathrm{RC}}(\epsilon)=\frac{\alpha^{2}}{4} \frac{g_{i}}{g_{j}} \frac{(\epsilon+I)^{2}}{\epsilon} \sigma_{\mathrm{PI}}
$$

where $\alpha$ is the fine-structure constant, $\epsilon$ is the photoelectron energy, $g_{j}$ is the statistical weight factor of the recombined ion, and $I$ is the ionization potential. Values of $\sigma_{\mathrm{RC}}$ are computed from the photoionization cross sections at a sufficiently large number of energies to delineate the nonresonant background and the autoionizing resonances, thereby representing both the radiative and the dielectronic recombination ( $R R$ and $D R$ ) processes. In the unified treatment the photoionization cross sections, $\sigma_{\mathrm{PI}}$, of a large number of bound states (group A) - all possible states with $n \leq n_{0} \sim 10$ - are obtained as described above. It is assumed that the recombining ion is in the ground state, and recombination can take place into the ground or any of the excited recombined $(e+$ ion) states.

Recombination rate coefficients of individual recombined levels are obtained by convolving recombination cross sections over the Maxwellian electron distribution $f(v)$ at a given temperature as,

$$
\alpha_{\mathrm{RC}}(T)=\int_{0}^{\infty} v f(v) \sigma_{\mathrm{RC}} d v
$$

Contributions from the low- $n$ group A bound levels are added for the total recombination rate coefficient, $\alpha_{\mathrm{RC}}$, and for the total recombination cross sections, $\sigma_{\mathrm{RC}}$.

Group B levels, $n_{0}<n \leq \infty$, are treated through the quantum defect theory of DR within the close-coupling approximation (Nahar \& Pradhan 1992, 1994). A generally valid approximation made in recombination to group B levels is that the background contribution is negligible, and DR is the dominant process in the region below the threshold of convergence for high- $n$ resonances. To each excited threshold of the core, $J_{i} \pi_{i}$, belongs an infinite series of $(N+1)$-electron levels, $J_{i} \pi_{i} \nu \ell$, to which recombination can occur. For the high $\nu$ levels, DR dominates while the background RR is small, and, hence, DR dominates the total recombination. The contributions from these levels are added by calculating the DR collision strengths, $\Omega_{\mathrm{DR}}$, an extension (Nahar \& Pradhan 1994) of the precise theory of radiation damping by Bell \& Seaton (1985):

$$
\Omega(\mathrm{DR})=\sum_{S L \pi} \sum_{n}(1 / 2)(2 S+1)(2 L+1) P_{n}^{S L \pi}
$$

where $P_{n}^{S L \pi}$ is the DR probability in entrance channel $n$, obtained as $P_{n}^{S L \pi}(\mathrm{DR})=\left(1-\boldsymbol{S}_{e e}^{\dagger} \boldsymbol{S}_{e e}\right)_{n}$, where $\boldsymbol{S}_{e e}$ is the matrix for 
TABLE 1

Ion Core Levels in the Eigenfunction Expansions of Ni xxvi and Ni xxvi

\begin{tabular}{|c|c|c|c|c|}
\hline \multirow[b]{2}{*}{ NuMber } & \multicolumn{2}{|c|}{ Ni xxVII } & \multicolumn{2}{|c|}{ Ni XxVIII } \\
\hline & Level & $\begin{array}{c}E_{t} \\
(\mathrm{ryd})\end{array}$ & Level & $\begin{array}{c}E_{t} \\
\text { (ryd) }\end{array}$ \\
\hline $1 \ldots \ldots \ldots$ & $1 s^{2}\left({ }^{1} S_{0}\right)$ & 0.0 & $1 s\left({ }^{2} S_{1 / 2}\right)$ & 0.00 \\
\hline . & $1 s 2 s\left({ }^{3} S_{1}\right)$ & 568.2566 & $2 p\left({ }^{2} P_{1 / 2}^{o}\right)$ & 593.654 \\
\hline $3 \ldots \ldots \ldots \ldots \ldots \ldots$ & $1 s 2 p\left({ }^{3} P_{0}^{o}\right)$ & 570.5865 & $2 s\left({ }^{2} S_{1 / 2}\right)$ & 593.686 \\
\hline . & $1 s 2 s\left({ }^{1} S_{0}\right)$ & 570.792 & $2 p\left({ }^{2} P_{3 / 2}^{o}\right)$ & 595.723 \\
\hline$\ldots$ & $1 s 2 p\left({ }^{3} P_{1}^{o}\right)$ & 570.7938 & $3 p\left({ }^{2} P_{1 / 2}^{o}\right)$ & 704.226 \\
\hline $6 \ldots \ldots \ldots \ldots \ldots$ & $1 s 2 p\left({ }^{3} P_{2}^{o}\right)$ & 572.2873 & $3 s\left({ }^{2} S_{1 / 2}\right)$ & 704.238 \\
\hline 7 ................... & $1 s 2 p\left({ }^{1} P_{1}^{o}\right)$ & 573.6669 & $3 d\left({ }^{2} D_{3 / 2}\right)$ & 704.821 \\
\hline $8 \ldots \ldots \ldots \ldots \ldots$ & $1 s 3 s\left({ }^{3} S_{1}\right)$ & 673.4568 & $3 p\left({ }^{2} P_{3 / 2}^{o}\right)$ & 704.823 \\
\hline . & $1 s 3 p\left({ }^{3} P_{0}\right)$ & 674.1039 & $3 d\left({ }^{2} D_{5 / 2}\right)$ & 705.024 \\
\hline 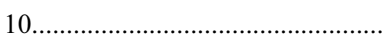 & $1 s 3 s\left({ }^{1} S_{0}\right)$ & 674.1223 & $4 p\left({ }^{2} P_{1 / 2}^{o}\right)$ & 742.836 \\
\hline 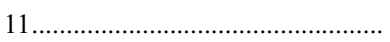 & $1 s 3 p\left({ }^{3} P_{1}^{o}\right)$ & 674.1493 & $4 s\left({ }^{2} S_{1 / 2}\right)$ & 742.841 \\
\hline $12 \ldots \ldots \ldots \ldots \ldots$ & $1 s 3 p\left({ }^{3} P_{2}^{o}\right)$ & 674.5945 & $4 d\left({ }^{2} D_{3 / 2}\right)$ & 743.065 \\
\hline 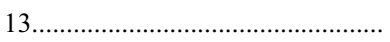 & $1 s 3 p\left({ }^{1} P_{1}^{o}\right)$ & 674.9808 & $4 p\left({ }^{2} P_{3 / 2}^{o}\right)$ & 743.078 \\
\hline 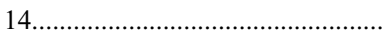 & $1 s 3 d\left({ }^{3} D_{1}\right)$ & 676.624 & $4 d\left({ }^{2} D_{5 / 2}\right)$ & 743.148 \\
\hline 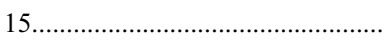 & $1 s 3 d\left({ }^{3} D_{2}\right)$ & 676.632 & $4 f\left({ }^{2} F_{5 / 2}^{o}\right)$ & 743.162 \\
\hline 16 & $1 s 3 d\left({ }^{1} D_{3}\right)$ & 676.806 & $4 f\left({ }^{2} F_{7 / 2}^{o}\right)$ & 743.205 \\
\hline 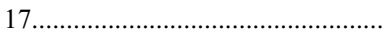 & $1 s 3 d\left({ }^{1} D_{2}\right)$ & 676.819 & & \\
\hline
\end{tabular}

Notes.-Ni xxviI: Correlations $2 s^{2}, 2 p^{2}, 3 s^{2}, 3 p^{2}, 3 d^{2}, 2 s 2 p, 2 s 3 s, 2 s 3 p, 2 s 3 d, 2 s 4 s, 2 s 4 p, 2 p 3 s$, $2 p 3 p, 2 p 3 d, 2 p 4 s$, and $2 p 4 p$. Ni xxVII: $\lambda_{n l} 1.0$, for $1 s$ to $4 p$. Ni XxVIII: No correlations; $\lambda_{n l} 1.0$, for $1 s$ to $4 f$.

electron scattering including radiation damping. The recombination cross section, $\sigma_{\mathrm{RC}}$, in megabarns $(\mathrm{Mb})$, is related to the collision strength, $\Omega_{\mathrm{RC}}$, as

$$
\sigma_{\mathrm{RC}}(i \rightarrow j)(\mathrm{Mb})=\pi \Omega_{\mathrm{RC}}(i, j) /\left(g_{i} k_{i}^{2}\right)\left(a_{0}^{2} / 1 . \times 10^{-18}\right),
$$

where $k_{i}^{2}$ is the incident electron energy in ryd. Since $\sigma_{\mathrm{RC}}$ diverges at zero electron energy, the total collision strength, $\Omega$, is used in the recombination rate calculations.

The group A levels in the unified method may not be necessarily restricted up to $n=10$. It can readily be extended to higher $n$. However, detailed calculations for photoionization cross sections of high- $n(>10)$ levels is unnecessary since they approach hydrogenic behavior. Background photoionization cross sections for these high- $n$ group B levels are computed hydrogenically to contribute to the total recombination rate. This high- $n$ background contribution is referred to as the "high- $n$ top-up" contribution (Nahar 1996).

\section{COMPUTATIONS}

The calculations for photoionization and electron-ion recombination span several stages of computation starting with obtaining the target (core ion) wavefunction through configuration interaction atomic structure calculations. The wavefunction expansion for Ni XXVI consists of 17 fine-structure levels of configurations $1 s^{2}, 1 s 2 s, 1 s 2 p, 1 s 3 s, 1 s 3 p$, and $1 s 3 d$ of target Ni xxvII. The levels, along with their relative energies, are given in Table 1. The set of correlation configurations in the atomic structure calculations are also given in Table 1 . The orbital wavefunctions are obtained from the atomic structure code SUPERSTRUCTURE (Eissner et al. 1974). The ThomasFermi scaling parameter $\left(\lambda_{\mathrm{nl}}\right)$ for each orbital is taken to be 1 . The second term of the wavefunction in equation (3), which contains bound state correlation functions for Ni XXVI, includes all possible $(N+1)$-particle configurations from 0 to maximum orbital occupancies as $2 s^{2}, 2 p^{2}, 3 s^{2}, 3 p^{2}, 3 d^{2}, 4 s$, and $4 p$. The energies in Table 1 are observed values from NIST. ${ }^{1}$ Radial integrals for the partial wave expansion in equation (3) are specified for orbitals $0 \leq \ell \leq 9$, with an $\boldsymbol{R}$-matrix basis set of 40 "continuum" functions for Ni xxvI. Computations are carried out for all angular momenta, $0 \leq L \leq 11,1 / 2 \leq J \leq 17 / 2$ for Ni XXvi.

The wavefunction expansion of $\mathrm{Ni}$ XXVII consists of 16 finestructure levels of configurations, $1 s, 2 s, 2 p, 3 s, 3 p, 3 d, 4 s, 4 p$, $4 d$, and $4 f$ of Ni xxvIII, as given in Table 1 . There are no correlation configurations, and the scaling parameter for each orbital is taken to be unity. The orbital wavefunctions and level energies are obtained from SUPERSTRUCTURE. The energies in Table 1 are the calculated ones. The bound state correlation functions, in the second term of the wavefunction, include all configurations from 0 to maximum orbital occupancies: $1 s^{2}$, $2 s^{2}, 2 p^{2}, 3 s^{2}, 3 p^{2}, 3 d^{2}, 4 s^{2}$, and $4 p^{2}, 4 d$, and $4 f$. Radial integrals for the partial wave expansion are specified for orbitals $0 \leq$ $\ell \leq 9$, with an $\boldsymbol{R}$-matrix basis set of 30 "continuum" functions for Ni XXVII. Computations are carried out for all angular momenta, $0 \leq L \leq 14,0 \leq J \leq 10$ for Ni XXVII.

Relativistic BPRM calculations in intermediate coupling are carried out in the close-coupling approximation using the $\boldsymbol{R}$-matrix package of codes (Berrington et al. 1995). These are extensions of the Opacity Project codes (Berrington et al. 1987) to include relativistic effects (Scott \& Burke 1980; Scott \& Taylor 1982; Berrington et al. 1995), implemented under the Iron Project (Hummer et al. 1993). The energy levels were identified using the code PRCBPID (Nahar \& Pradhan 2000).

Both the partial and the total photoionization cross sections are obtained for all bound levels. Coupled channel calculations for $\sigma_{\mathrm{PI}}$ include both the background and the resonance structures (owing to the doubly excited autoionizing states) in the cross sections. Radiation damping of resonances up to $n=10$ are included through use of the extended codes STGF and STGBF (Nahar \& Pradhan 1994; Zhang et al. 1999). The

\footnotetext{
${ }^{1}$ See the NIST Web site, http://www.nist.gov.
} 
TABLE 2

Radiative Decay Rates, $A_{j i}$, IN s ${ }^{-1}$ for Allowed Transitions To THE Ground LeVel, $1 s^{2}{ }^{1} S_{0}$ FOR Ni XXVII AND $1 s{ }^{2} S_{1 / 2}$ FOR Ni XXVIII

\begin{tabular}{|c|c|}
\hline Target State & $\begin{array}{c}A_{f i} \\
\left(\mathrm{~s}^{-1}\right)\end{array}$ \\
\hline \multicolumn{2}{|l|}{ Ni xxvII: GD- $1 s^{2}{ }^{1} S_{0}$} \\
\hline 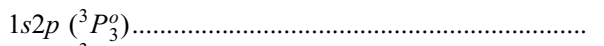 & $7.21(13)$ \\
\hline 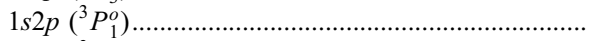 & $6.23(14)$ \\
\hline $1 s 3 p\left({ }^{3} P_{3}^{o}\right) \ldots \ldots$ & $2.17(13)$ \\
\hline 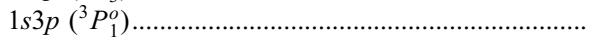 & $1.80(14)$ \\
\hline \multicolumn{2}{|l|}{ Ni xxvIII: GD- $1 s^{2} S^{1 / 2}$} \\
\hline $2 p\left({ }^{2} P_{1 / 2}^{o}\right) \ldots \ldots$ & $3.79(14)$ \\
\hline $2 p\left({ }^{2} P_{3 / 2}^{o}\right) \ldots \ldots \ldots$ & $3.80(14)$ \\
\hline 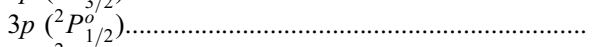 & $9.34(13)$ \\
\hline $3 p\left({ }^{2} P_{3 / 2}^{o}\right) \ldots \ldots \ldots$ & $9.73(13)$ \\
\hline 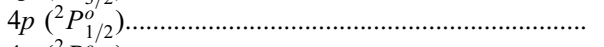 & $3.23(13)$ \\
\hline 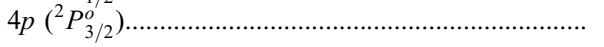 & $3.50(13)$ \\
\hline
\end{tabular}

BPRM calculations are carried out for each total angular momentum symmetry $J \pi$, corresponding to a set of fine-structure target levels $J_{t}$. Radiation damping of resonances within the close-coupling BPRM calculations are described in Zhang et al. (1999 and references therein). The program PBPRAD is used to extend the total photoionization cross sections in the highenergy region, beyond the highest target threshold in the closecoupling wavefunction expansion of the ion, by a "tail" using a fitting formula as well as the Kramers formula $\sigma(E)=$ $\sigma_{\mathrm{PI}}^{0}\left(E^{0} / E\right)^{3}$, where $\sigma^{0}$ is the last tabulated cross section at energy $E^{0}$ above all target thresholds, as described in Nahar \& Pradhan (1994).

In the higher energy region, $\nu_{0}<\nu \leq \infty$, below each target threshold where the resonances are narrow and dense and the background is negligible, we compute detailed and resonance averaged DR cross sections (Bell \& Seaton 1985; Nahar \& Pradhan 1994). The radiative decay probabilities used for the ions are given in Table 2. The DR collision strengths in the BPRM approximation are obtained using extensions of the $\boldsymbol{R}$-matrix asymptotic region code STGF (Nahar \& Pradhan 1994; Zhang et al. 1999). It is necessary to use a very fine energy mesh in order to delineate the resonance structures.

Level-specific recombination cross sections, $\sigma_{\mathrm{RC}}(i)$, into various bound levels $i \equiv n(S L J)$ of the recombined ( $e+$ ion) system, are obtained from partial photoionization cross sections $\sigma_{\mathrm{PI}}(i, g)$ of the level $i$ into the ground level $g$ of the recombining ion. These detailed photorecombination cross sections are calculated in the energy region from the threshold energy up to $E\left(\nu=\nu_{0} \approx 10.0\right)$, where $\nu$ is the effective quantum number relative to the target level of the recombining ion. The resonances up to $\nu \leq \nu_{0}$ are delineated with a fine energy mesh. The electrons in this energy range generally recombine to a large number of final $(e+$ ion) levels. The level-specific rates are obtained for energies going up to infinity. Recombination cross sections are computed for all coupled symmetries and levels, and summed to obtain the total $\sigma_{\mathrm{RC}}$ using the program RECXS (Nahar et al. 2000).

The program RECXS sums up the level-specific rates for the total recombination rates, which also includes the contributions from the resonant high- $n$ DR of $\nu_{0}<\nu<\infty$. As an additional check on the numerical calculations, the total recombination rate coefficients, $\alpha_{\mathrm{RC}}$, are also calculated from the
TABLE 3

Total Recombination Rate Coefficients $\alpha_{\text {RC }}(T)$ FOR Ni XXVI, Ni XXVII, AND Ni XXVIII

\begin{tabular}{|c|c|c|c|}
\hline \multirow{2}{*}{$\begin{array}{c}\log _{10} T \\
(\mathrm{~K})\end{array}$} & \multicolumn{3}{|c|}{$\alpha_{\mathrm{RC}}\left(\mathrm{cm}^{3} \mathrm{~s}^{-1}\right)$} \\
\hline & Ni xxvi & Ni XxvII & Ni XxVIII \\
\hline 1.0 & $2.86 \mathrm{E}-08$ & $3.28 \mathrm{E}-08$ & $3.72 \mathrm{E}-08$ \\
\hline $1.1 \ldots$ & $2.54 \mathrm{E}-08$ & $2.92 \mathrm{E}-08$ & $3.31 \mathrm{E}-08$ \\
\hline $1.2 \ldots$ & $2.26 \mathrm{E}-08$ & $2.60 \mathrm{E}-08$ & $2.95 \mathrm{E}-08$ \\
\hline $1.3 \ldots \ldots$ & $2.01 \mathrm{E}-08$ & $2.31 \mathrm{E}-08$ & $2.62 \mathrm{E}-08$ \\
\hline 1.4 & $1.78 \mathrm{E}-08$ & $2.05 \mathrm{E}-08$ & $2.33 \mathrm{E}-08$ \\
\hline $1.5 \ldots \ldots \ldots$ & $1.58 \mathrm{E}-08$ & $1.82 \mathrm{E}-08$ & $2.07 \mathrm{E}-08$ \\
\hline $1.6 \ldots \ldots \ldots+\ldots$ & $1.40 \mathrm{E}-08$ & $1.62 \mathrm{E}-08$ & $1.84 \mathrm{E}-08$ \\
\hline 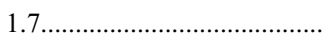 & $1.24 \mathrm{E}-08$ & $1.43 \mathrm{E}-08$ & $1.63 \mathrm{E}-08$ \\
\hline $1.8 \ldots$ & $1.10 \mathrm{E}-08$ & $1.27 \mathrm{E}-08$ & $1.44 \mathrm{E}-08$ \\
\hline $1.9 \ldots \ldots$ & $9.74 \mathrm{E}-09$ & $1.12 \mathrm{E}-08$ & $1.28 \mathrm{E}-08$ \\
\hline $2.0 \ldots \ldots \ldots \ldots \ldots$ & $8.60 \mathrm{E}-09$ & $9.94 \mathrm{E}-09$ & $1.13 \mathrm{E}-08$ \\
\hline $2.1 \ldots \ldots$ & $7.60 \mathrm{E}-09$ & $8.78 \mathrm{E}-09$ & $1.00 \mathrm{E}-08$ \\
\hline $2.2 \ldots \ldots \ldots$ & $6.70 \mathrm{E}-09$ & $7.76 \mathrm{E}-09$ & $8.85 \mathrm{E}-09$ \\
\hline $2.3 \ldots \ldots \ldots \ldots$ & $5.90 \mathrm{E}-09$ & $6.84 \mathrm{E}-09$ & $7.81 \mathrm{E}-09$ \\
\hline $2.4 \ldots \ldots \ldots$ & $5.20 \mathrm{E}-09$ & $6.03 \mathrm{E}-09$ & $6.89 \mathrm{E}-09$ \\
\hline $2.5 \ldots \ldots \ldots$ & $4.58 \mathrm{E}-09$ & $5.31 \mathrm{E}-09$ & $6.07 \mathrm{E}-09$ \\
\hline $2.6 \ldots \ldots$ & $4.01 \mathrm{E}-09$ & $4.67 \mathrm{E}-09$ & $5.35 \mathrm{E}-09$ \\
\hline $2.7 \ldots \ldots \ldots$ & $3.52 \mathrm{E}-09$ & $4.10 \mathrm{E}-09$ & $4.70 \mathrm{E}-09$ \\
\hline $2.8 \ldots \ldots$ & $3.09 \mathrm{E}-09$ & $3.60 \mathrm{E}-09$ & $4.14 \mathrm{E}-09$ \\
\hline $2.9 \ldots \ldots$. & $2.71 \mathrm{E}-09$ & $3.16 \mathrm{E}-09$ & $3.63 \mathrm{E}-09$ \\
\hline 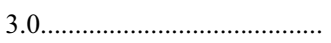 & $2.37 \mathrm{E}-09$ & $2.77 \mathrm{E}-09$ & $3.19 \mathrm{E}-09$ \\
\hline 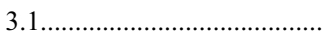 & $2.07 \mathrm{E}-09$ & $2.43 \mathrm{E}-09$ & $2.80 \mathrm{E}-09$ \\
\hline 3.2 & $1.81 \mathrm{E}-09$ & $2.13 \mathrm{E}-09$ & $2.45 \mathrm{E}-09$ \\
\hline 3.3 & $1.58 \mathrm{E}-09$ & $1.86 \mathrm{E}-09$ & $2.15 \mathrm{E}-09$ \\
\hline 3.4 & $1.38 \mathrm{E}-09$ & $1.63 \mathrm{E}-09$ & $1.88 \mathrm{E}-09$ \\
\hline $3.5 \ldots \ldots \ldots$ & $1.21 \mathrm{E}-09$ & $1.42 \mathrm{E}-09$ & $1.65 \mathrm{E}-09$ \\
\hline 3.6. & $1.05 \mathrm{E}-09$ & $1.24 \mathrm{E}-09$ & $1.44 \mathrm{E}-09$ \\
\hline 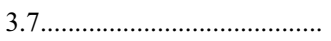 & $9.14 \mathrm{E}-10$ & $1.08 \mathrm{E}-09$ & $1.26 \mathrm{E}-09$ \\
\hline 3.8. & $7.95 \mathrm{E}-10$ & $9.44 \mathrm{E}-10$ & $1.10 \mathrm{E}-09$ \\
\hline 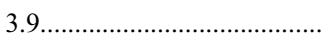 & $6.91 \mathrm{E}-10$ & $8.22 \mathrm{E}-10$ & $9.59 \mathrm{E}-10$ \\
\hline $4.0 \ldots \ldots \ldots$ & $6.00 \mathrm{E}-10$ & $7.16 \mathrm{E}-10$ & $8.37 \mathrm{E}-10$ \\
\hline $4.1 \ldots \ldots \ldots$ & $5.21 \mathrm{E}-10$ & $6.23 \mathrm{E}-10$ & $7.29 \mathrm{E}-10$ \\
\hline $4.2 \ldots \ldots \ldots$ & $4.52 \mathrm{E}-10$ & $5.42 \mathrm{E}-10$ & $6.37 \mathrm{E}-10$ \\
\hline 4.3 & $3.92 \mathrm{E}-10$ & $4.71 \mathrm{E}-10$ & $5.54 \mathrm{E}-10$ \\
\hline $4.4 \ldots \ldots \ldots$ & $3.39 \mathrm{E}-10$ & $4.09 \mathrm{E}-10$ & $4.82 \mathrm{E}-10$ \\
\hline 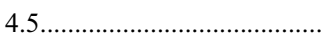 & $2.94 \mathrm{E}-10$ & $3.55 \mathrm{E}-10$ & $4.20 \mathrm{E}-10$ \\
\hline 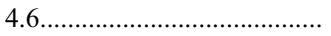 & $2.54 \mathrm{E}-10$ & $3.08 \mathrm{E}-10$ & $3.65 \mathrm{E}-10$ \\
\hline ……………… & $2.19 \mathrm{E}-10$ & $2.67 \mathrm{E}-10$ & $3.17 \mathrm{E}-10$ \\
\hline$\ldots$ & $1.89 \mathrm{E}-10$ & $2.32 \mathrm{E}-10$ & $2.76 \mathrm{E}-10$ \\
\hline 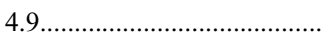 & $1.63 \mathrm{E}-10$ & $2.00 \mathrm{E}-10$ & $2.39 \mathrm{E}-10$ \\
\hline .................. & $1.41 \mathrm{E}-10$ & $1.73 \mathrm{E}-10$ & $2.08 \mathrm{E}-10$ \\
\hline ............. & $1.21 \mathrm{E}-10$ & $1.50 \mathrm{E}-10$ & $1.80 \mathrm{E}-10$ \\
\hline$\ldots \ldots \ldots \ldots . . .$. & $1.04 \mathrm{E}-10$ & $1.29 \mathrm{E}-10$ & $1.56 \mathrm{E}-10$ \\
\hline 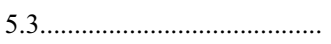 & $8.92 \mathrm{E}-11$ & $1.11 \mathrm{E}-10$ & $1.35 \mathrm{E}-10$ \\
\hline 5.4 & $7.65 \mathrm{E}-11$ & $9.61 \mathrm{E}-11$ & $1.17 \mathrm{E}-10$ \\
\hline 5.5 & $6.55 \mathrm{E}-11$ & $8.27 \mathrm{E}-11$ & $1.01 \mathrm{E}-10$ \\
\hline 5.6 & $5.59 \mathrm{E}-11$ & $7.12 \mathrm{E}-11$ & $8.69 \mathrm{E}-11$ \\
\hline 5.7 & $4.77 \mathrm{E}-11$ & $6.11 \mathrm{E}-11$ & $7.50 \mathrm{E}-11$ \\
\hline 5.8 & $4.07 \mathrm{E}-11$ & $5.24 \mathrm{E}-11$ & $6.46 \mathrm{E}-11$ \\
\hline $5.9 \ldots \ldots \ldots \ldots$ & $3.46 \mathrm{E}-11$ & $4.49 \mathrm{E}-11$ & $5.56 \mathrm{E}-11$ \\
\hline $6.0 \ldots$ & $2.93 \mathrm{E}-11$ & $3.84 \mathrm{E}-11$ & $4.78 \mathrm{E}-11$ \\
\hline $6.1 \ldots$ & $2.48 \mathrm{E}-11$ & $3.28 \mathrm{E}-11$ & $4.10 \mathrm{E}-11$ \\
\hline $6.2 \ldots$ & $2.10 \mathrm{E}-11$ & $2.80 \mathrm{E}-11$ & $3.53 \mathrm{E}-11$ \\
\hline $6.3 \ldots \ldots \ldots$ & $1.77 \mathrm{E}-11$ & $2.39 \mathrm{E}-11$ & $3.02 \mathrm{E}-11$ \\
\hline 6.4 & $1.49 \mathrm{E}-11$ & $2.03 \mathrm{E}-11$ & $2.58 \mathrm{E}-11$ \\
\hline 6.5 & $1.25 \mathrm{E}-11$ & $1.72 \mathrm{E}-11$ & $2.21 \mathrm{E}-11$ \\
\hline 6.6 & $1.05 \mathrm{E}-11$ & $1.46 \mathrm{E}-11$ & $1.88 \mathrm{E}-11$ \\
\hline 6.7 & $8.72 \mathrm{E}-12$ & $1.23 \mathrm{E}-11$ & $1.60 \mathrm{E}-11$ \\
\hline 6.8 & $7.26 \mathrm{E}-12$ & $1.04 \mathrm{E}-11$ & $1.36 \mathrm{E}-11$ \\
\hline 6.9. & $6.03 \mathrm{E}-12$ & $8.75 \mathrm{E}-12$ & $1.16 \mathrm{E}-11$ \\
\hline 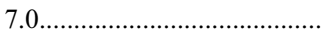 & $5.00 \mathrm{E}-12$ & $7.35 \mathrm{E}-12$ & $9.78 \mathrm{E}-12$ \\
\hline
\end{tabular}


TABLE 3-Continued

\begin{tabular}{|c|c|c|c|}
\hline \multirow{2}{*}{$\begin{array}{c}\log _{10} T \\
(\mathrm{~K})\end{array}$} & \multicolumn{3}{|c|}{$\alpha_{\mathrm{RC}}\left(\mathrm{cm}^{3} \mathrm{~s}^{-1}\right)$} \\
\hline & Ni xxvI & Ni XXVII & Ni XXVIII \\
\hline $7.1 \ldots$ & $4.17 \mathrm{E}-12$ & $6.18 \mathrm{E}-12$ & $8.25 \mathrm{E}-12$ \\
\hline ................ & $3.51 \mathrm{E}-12$ & $5.22 \mathrm{E}-12$ & $6.97 \mathrm{E}-12$ \\
\hline $7.3 \ldots$ & $3.01 \mathrm{E}-12$ & $4.44 \mathrm{E}-12$ & $5.85 \mathrm{E}-12$ \\
\hline $7.4 \ldots$ & $2.63 \mathrm{E}-12$ & $3.82 \mathrm{E}-12$ & $4.90 \mathrm{E}-12$ \\
\hline $7.5 \ldots \ldots$ & $2.32 \mathrm{E}-12$ & $3.30 \mathrm{E}-12$ & $4.10 \mathrm{E}-12$ \\
\hline $7.6 \ldots \ldots$ & $2.05 \mathrm{E}-12$ & $2.86 \mathrm{E}-12$ & $3.41 \mathrm{E}-12$ \\
\hline $7.7 \ldots \ldots \ldots$ & $1.78 \mathrm{E}-12$ & $2.47 \mathrm{E}-12$ & $2.83 \mathrm{E}-12$ \\
\hline $7.8 \ldots \ldots$ & $1.53 \mathrm{E}-12$ & $2.10 \mathrm{E}-12$ & $2.34 \mathrm{E}-12$ \\
\hline $7.9 \ldots$ & $1.28 \mathrm{E}-12$ & $1.77 \mathrm{E}-12$ & $1.92 \mathrm{E}-12$ \\
\hline $8.0 \ldots \ldots \ldots$ & $1.06 \mathrm{E}-12$ & $1.46 \mathrm{E}-12$ & $1.58 \mathrm{E}-12$ \\
\hline $8.1 \ldots \ldots \ldots$ & $8.52 \mathrm{E}-13$ & $1.19 \mathrm{E}-12$ & $1.28 \mathrm{E}-12$ \\
\hline $8.2 \ldots$ & $6.76 \mathrm{E}-13$ & $9.60 \mathrm{E}-13$ & $1.04 \mathrm{E}-12$ \\
\hline $8.3 \ldots .$. & $5.29 \mathrm{E}-13$ & $7.64 \mathrm{E}-13$ & $8.36 \mathrm{E}-13$ \\
\hline $8.4 \ldots \ldots \ldots \ldots . . . . .$. & $4.09 \mathrm{E}-13$ & $6.01 \mathrm{E}-13$ & $6.70 \mathrm{E}-13$ \\
\hline $8.5 \ldots \ldots \ldots \ldots .$. & $3.13 \mathrm{E}-13$ & $4.69 \mathrm{E}-13$ & $5.33 \mathrm{E}-13$ \\
\hline 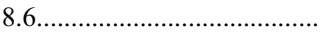 & $2.37 \mathrm{E}-13$ & $3.64 \mathrm{E}-13$ & $4.22 \mathrm{E}-13$ \\
\hline $8.7 \ldots \ldots \ldots \ldots \ldots$ & $1.79 \mathrm{E}-13$ & $2.80 \mathrm{E}-13$ & $3.32 \mathrm{E}-13$ \\
\hline 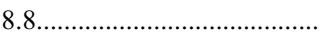 & $1.34 \mathrm{E}-13$ & $2.14 \mathrm{E}-13$ & $2.59 \mathrm{E}-13$ \\
\hline 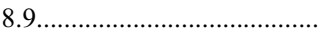 & $1.00 \mathrm{E}-13$ & $1.63 \mathrm{E}-13$ & $2.02 \mathrm{E}-13$ \\
\hline 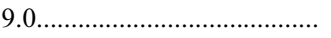 & $7.44 \mathrm{E}-14$ & $1.24 \mathrm{E}-13$ & $1.56 \mathrm{E}-13$ \\
\hline
\end{tabular}

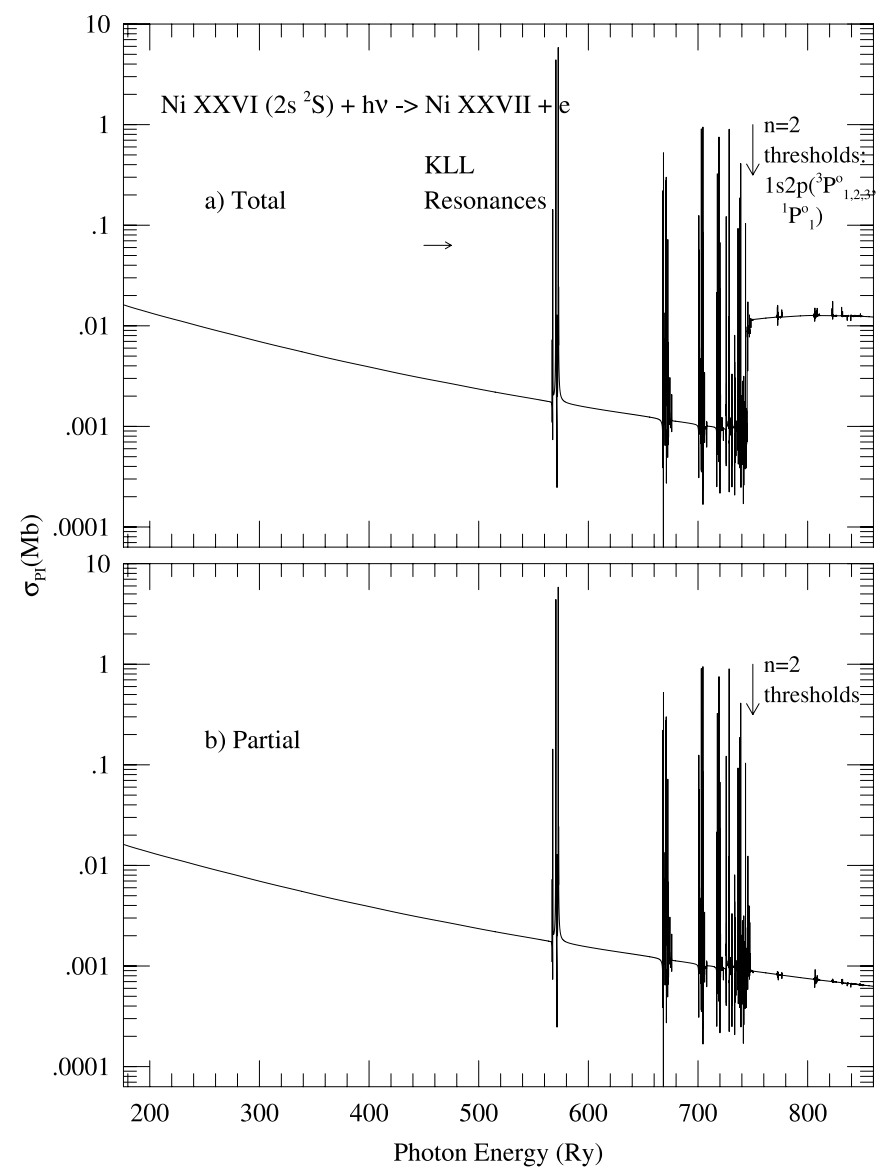

FIG. 1.-Photoionization cross sections $\left(\sigma_{\mathrm{PI}}\right)$ of the ground level $1 s^{2} 2 s\left({ }^{2} S_{1 / 2}\right)$ of Ni XxvI. (a) Total cross section; the large jump around $n=2$ thresholds $(\sim 735$ ryd $)$ is the K-shell ionization edge $\left(h \nu+1 s^{2} 2 l \rightarrow 1 s 2 l+e\right)$. (b) Partial cross section into the ground level $1 s^{2}\left({ }^{1} S_{0}\right)$ of Ni xxvII; note that the jump is no longer present and the cross section is continuous across the $n=2$ thresholds of Ni XXVII.

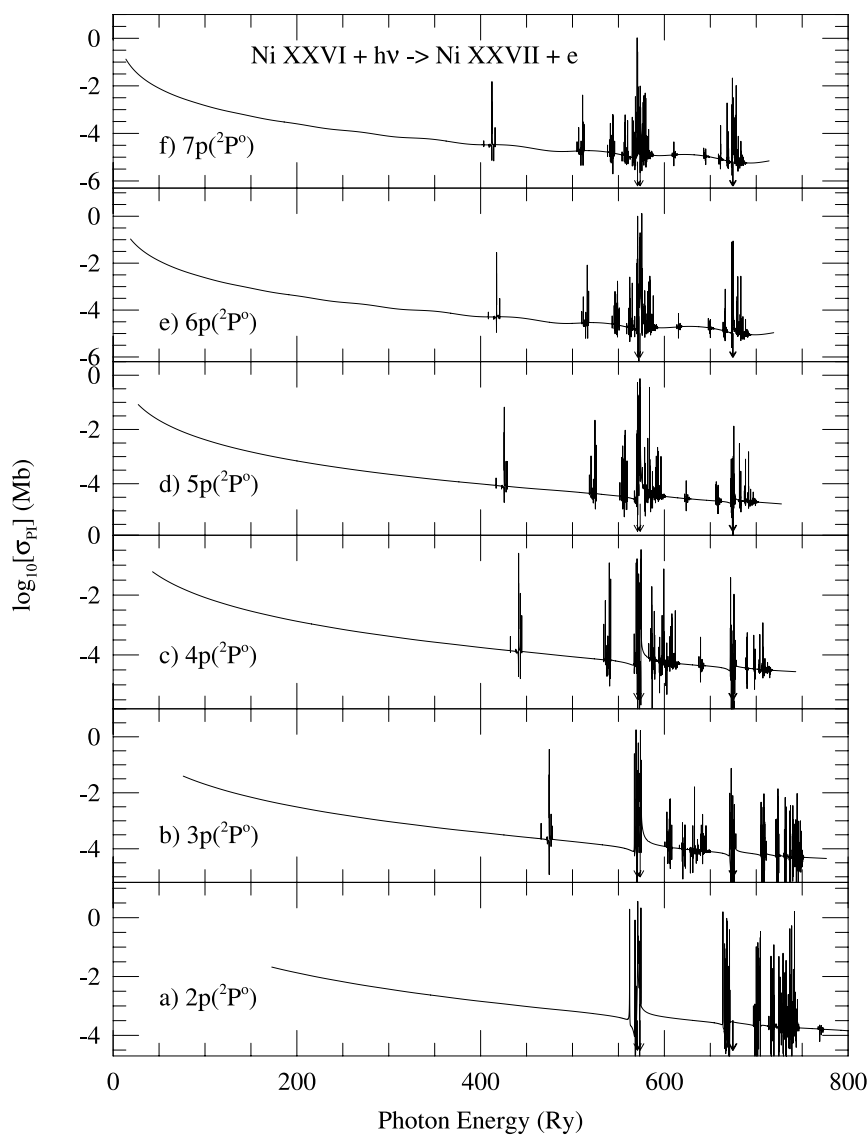

FIG. 2.-Partial photoionization cross sections of the Rydberg series of levels, $1 s^{2} n p\left({ }^{2} P_{1 / 2}^{o}\right)$ with $2 \leq n \leq 7$, of Ni XxvI into the ground level $1 s^{2}\left({ }^{1} S_{0}\right)$ of Ni XxvII. Prominent PEC (photoexcitation-of-core) resonances are seen (arrows) at about 571 ryd of excited core levels $1 s 2 p^{3} P_{1}^{o},{ }^{1} P_{1}^{o}$ and about 674 ryd of levels $1 s 3 p{ }^{3} P_{1}^{o},{ }^{1} P_{1}^{o}$.

total recombination collision strength, $\Omega_{\mathrm{RC}}$, obtained from all the photoionization cross sections, and the high- $n$ DR collision strengths. The agreement between the two numerical approaches is within a few percent.

The small background (nonresonant) contribution from the high- $n$ states $(10<n \leq \infty)$ to total recombination is included as the "top-up" part, computed in the hydrogenic approximation (Nahar 1996). This contribution is important at low temperatures as the recombination rate is dominated by the $R R$, but negligible at high temperatures.

\section{RESULTS AND DISCUSSION}

The inverse processes of photoionization and recombination of ( $\mathrm{Ni}$ xxVI $+h \nu \leftrightarrow \mathrm{Ni}$ XXVII $+e)$ and ( $\mathrm{Ni}$ XXVII $+h \nu \leftrightarrow$ $\mathrm{Ni}$ XXVIII $+e$ ) are studied in detail. Total recombination rate coefficients for the hydrogenic Ni XxVIII are also presented along with those of Ni xxVI and Ni XxVII in Table 3 for completeness.

Both the total and the partial photoionization cross sections, including the autoionizing resonances, are obtained for Ni XxvI and Ni XXVII for the first time and are available electronically. The total photoionization cross sections correspond to leaving the core ion in various excited states and are needed in astrophysical applications, such as in ionization balance calculations. The partial cross sections for leaving the core in the ground level are needed for applications such as recombination rate coefficients.

The total unified recombination collision strengths $\left(\Omega_{\mathrm{RC}}\right)$, cross sections $\left(\sigma_{\mathrm{RC}}\right)$, and recombination rate $\left[\alpha_{\mathrm{RC}}(E)\right]$ with electron energies are presented for $\mathrm{Ni}$ xxvI and $\mathrm{Ni}$ XxVII, and the 

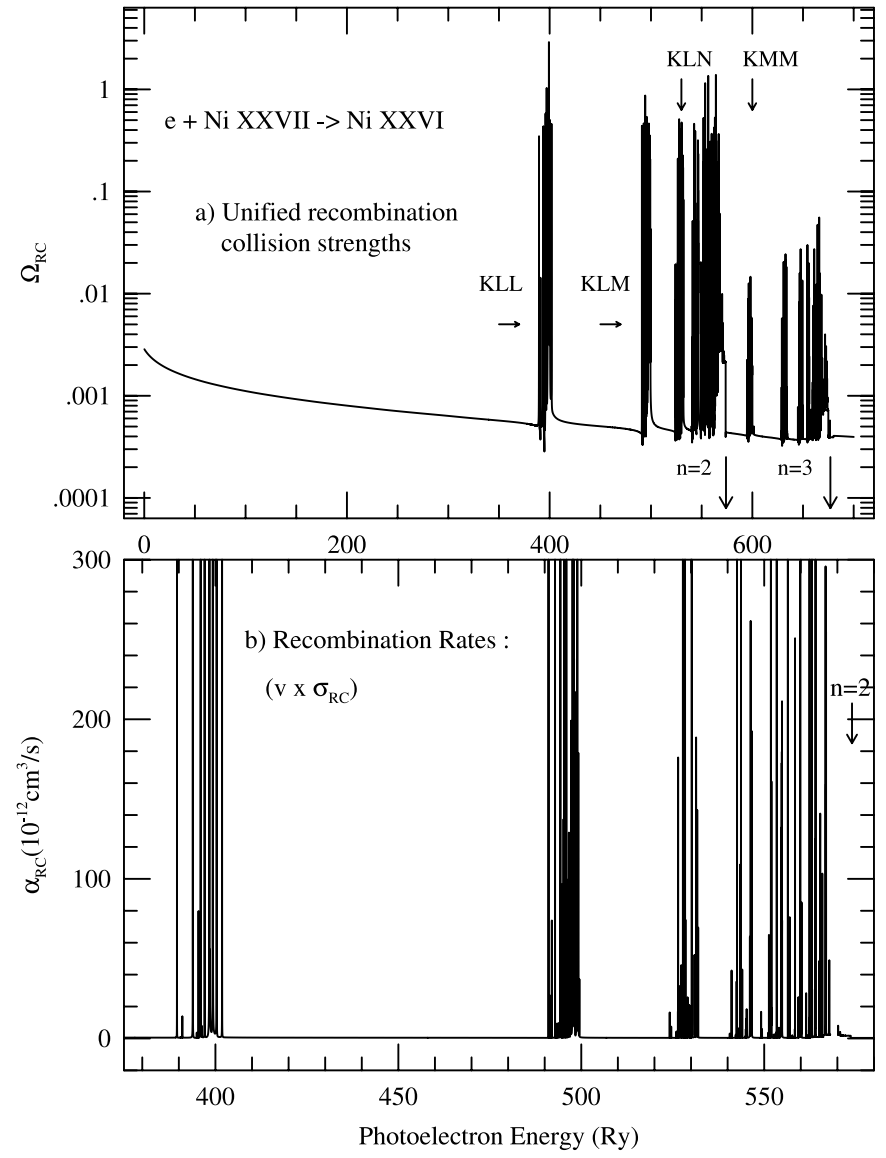

FIG. 3.- (a) Total unified ( $e+$ ion) recombination collision strengths, $\Omega_{\mathrm{RC}}$ and $(b)$ unified recombination rate coefficients, $\alpha_{\mathrm{RC}}(E)$, with photoelectron energy of Ni Xxvi. Note the separated resonance complexes, KLL, KLM, etc., of $n=2$ and $\mathrm{KMM}, \mathrm{KMN}$, etc., of $n=3$ thresholds. The quantity $\alpha_{\mathrm{RC}}(E)$, convolved with a bandwidth, is measurable.

data are available electronically. Total unified recombination rate coefficients, $\alpha_{\mathrm{RC}}(T)$, are computed in two different ways to enable numerical checks: (i) from the sum of the level-specific rate coefficients and the high- $n$ DR contribution and (ii) from total collision strengths calculated from photoionization cross sections directly and the DR contribution. The differences between the two are typically within a few percent, thus providing a numerical and self-consistency check particularly on the resolution of resonances.

Level-specific and total recombination rate coefficients, $\alpha_{i}(n S L J, n \leq 10)$ and $\alpha_{\mathrm{RC}}(T)$, respectively, are obtained using the BPRM unified treatment for Ni XXVI and Ni XXVII. Calculations of the recombination-cascade contributions for important lines require accurate atomic parameters for fine-structure levels up to fairly high $n$ levels, as reported here. Level-specific recombination rate coefficients of all bound levels and the summed total recombination rate coefficients $\left[\alpha_{\mathrm{RC}}(T)\right]$ recombined into an infinite number of bound states are presented. The level-specific rate coefficients are obtained for the first time. Existing data are available only for individual total RR and DR rates.

Important features in photoionization and electron-ion recombination for each ion are discussed separately below.

\subsection{Ni XXVI}

A total of 98 bound levels are found for Ni XXVI with $n \leq 10$, $0 \leq l \leq 9, \quad 0 \leq L \leq 11$, and total angular momentum of $1 / 2 \leq J \leq 17 / 2$ (Nahar 2002).

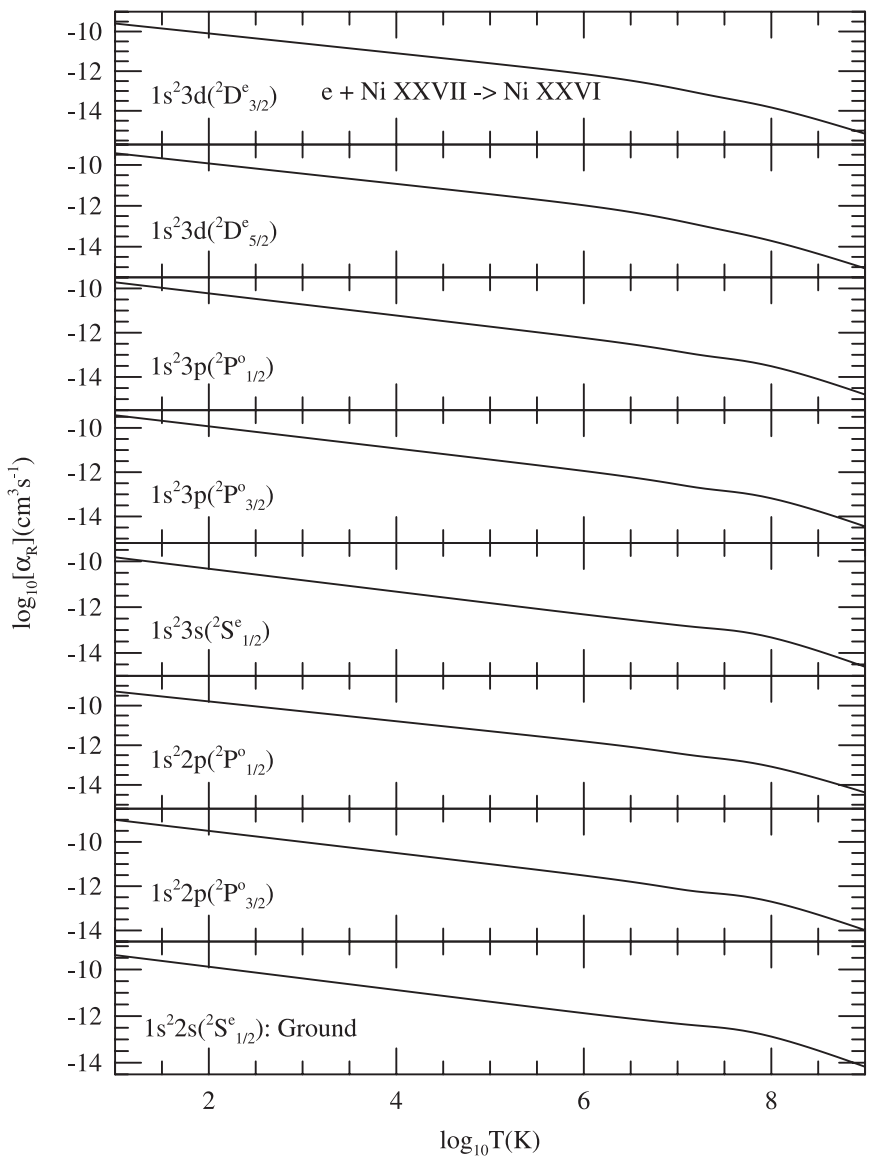

FIG. 4.-Level-specific recombination rate coefficients for Ni XxvI recombining to ground and excited $n=2,3$ levels.

\subsubsection{Photoionization}

Cross sections $\left(\sigma_{\mathrm{PI}}\right)$ for both total and partial photoionization are obtained for all 98 bound levels of Ni XXVI.

Figures $1 a$ and $1 b$ present the ground state photoionization cross section for Ni XXVI $\left(1 s^{2} 2 s^{2} S_{1 / 2}\right)$. Figure $1 a$ presents the total photoionization cross sections summed over the various target thresholds for ionization, and Figure $1 b$ presents the partial cross sections of the ground level into the ground $1 s^{2}\left({ }^{1} S_{0}\right)$ level of residual ion Ni XxvII. The resonances at high energies belong to the Rydberg series converging on to the $n=2,3$ levels of core Ni XxVII. These are the well-known KLL, KLN resonances, for example, as discussed by Nahar \& Pradhan (2003). Since the first excited levels of $n=2$ thresholds of the core ion Ni XXVII lie at high energies, the cross sections decrease monotonically over a large energy range before the Rydberg series of resonances appear. The total and the partial cross sections are identical below the first excited level of the residual ion beyond which total $\sigma_{\mathrm{PI}}$ increases because of added contributions from excited channels (Fig. 1). The distinct difference between the total and partial cross sections in Figure 1 comes from the contribution of channels with excited $n=2$ thresholds. The K-shell ionization jump at the $n=2$ target levels in total $\sigma_{\mathrm{PI}}$ is due to inner-shell photoionization:

$$
h \nu+\mathrm{Ni} \operatorname{xxVI}\left(1 s^{2} 2 s\right) \rightarrow e+\mathrm{Ni} \operatorname{xXVII}(1 s 2 s, 1 s 2 p)
$$

In X-ray photoionization models inner-shell edges play an important role in overall ionization rates. 


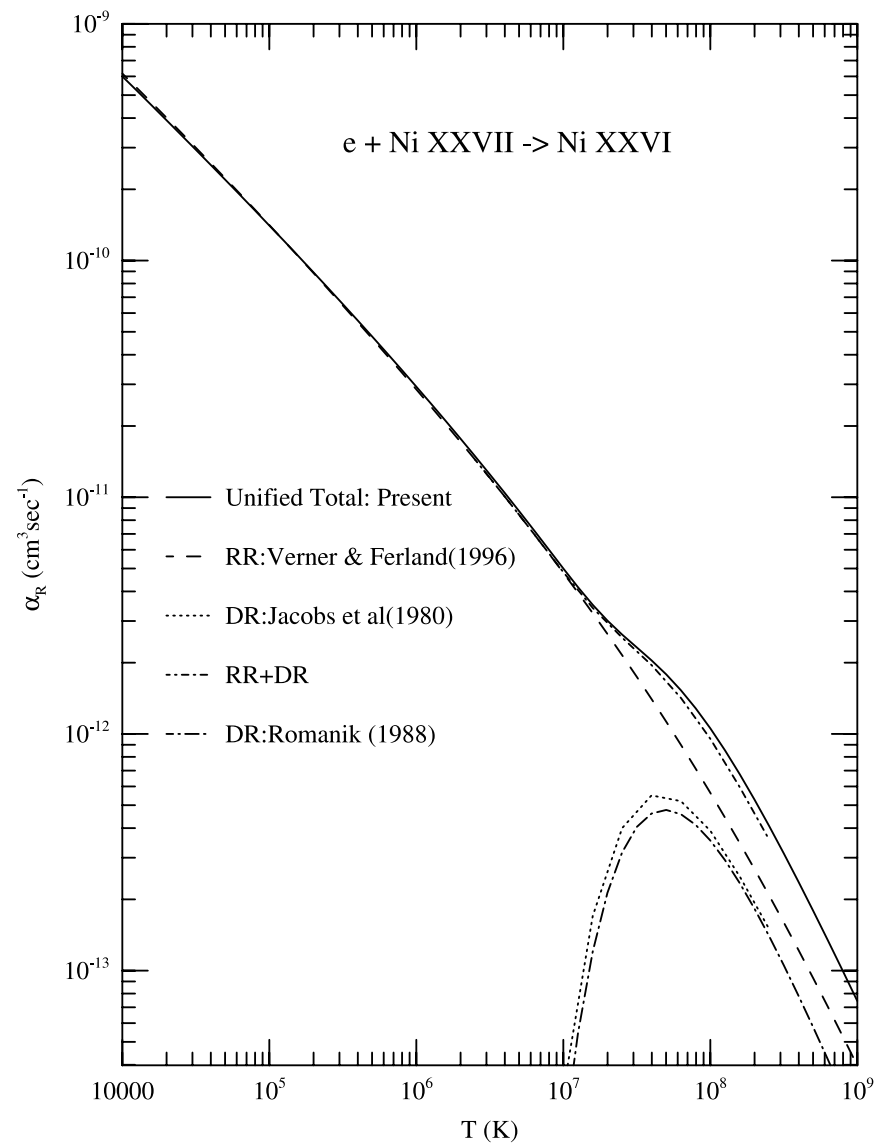

FIG. 5.-Total unified recombination rate coefficients, $\alpha_{\mathrm{RC}}(T)$, for Ni XXvI: total unified (solid line), RR rates (dashed line) by Verner \& Ferland (1996), DR rates (dotted line) by Jacobs et al. (1980) and DR rates (chain-dashed line) by Romanik (1988), and sum of RR + DR (dot-dashed line).

Figure 2 presents partial photoionization cross sections of excited Rydberg series of levels $1 s^{2} n p\left({ }^{2} P_{1 / 2}^{o}\right)$, with $2 \leq n \leq 7$, of Ni XXVI. The figure illustrates the resonant structures at higher energies. especially the photoexcitation-of-core (PEC) resonances at energies associated with dipole transitions in the core ion. PEC resonances in photoionization cross sections are seen in all excited bound levels of Ni XxvI, as in Figure 2, at photon energies 570.79, $573.67,674.15$, and 674.98 ryd owing to core excitations to levels $1 s 2 p\left({ }^{3} P_{1}^{o}\right), 1 s 2 p\left({ }^{1} P_{1}^{o}\right), 1 s 3 p\left({ }^{3} P_{1}^{o}\right)$, and $1 s 3 p\left({ }^{1} P_{1}^{o}\right)$ of Ni XXVII. At these energies the core ion goes through an allowed transition, while the outer electron remains as a "spectator" in a doubly excited resonant state, followed by autoionization into the ground level of the core. The effect is more prominent for cross sections of higher excited levels. These resonances depict the nonhydrogenic behavior of cross sections of excited levels and contribute to features in level-specific recombination rates.

\subsubsection{Electron-Ion Recombination}

The collision strength for electron-ion recombination, $\Omega_{\mathrm{RC}}$, is related to recombination cross sections, $\sigma_{\mathrm{RC}}$ (eq. [10]), and shows similar features. However, $\sigma_{\mathrm{RC}}$ blows up at zero electron energy. Hence, the total unified photorecombination collision strength, $\Omega_{\mathrm{RC}}$, for Ni XXVI is presented in Figure $3 a$. The strength $\Omega_{\mathrm{RC}}$, like photoionization cross sections $\sigma_{\mathrm{PI}}$, decays smoothly with energy, before the emergence of resonance complexes at high energies. However, $\Omega_{\mathrm{RC}}$ is more complex than a single level $\sigma_{\mathrm{PI}}$ as it is obtained from summed contributions of $\sigma_{\mathrm{PI}}$ of all levels. The resonance complexes in $\Omega_{\mathrm{RC}}$ (marked in the figure)

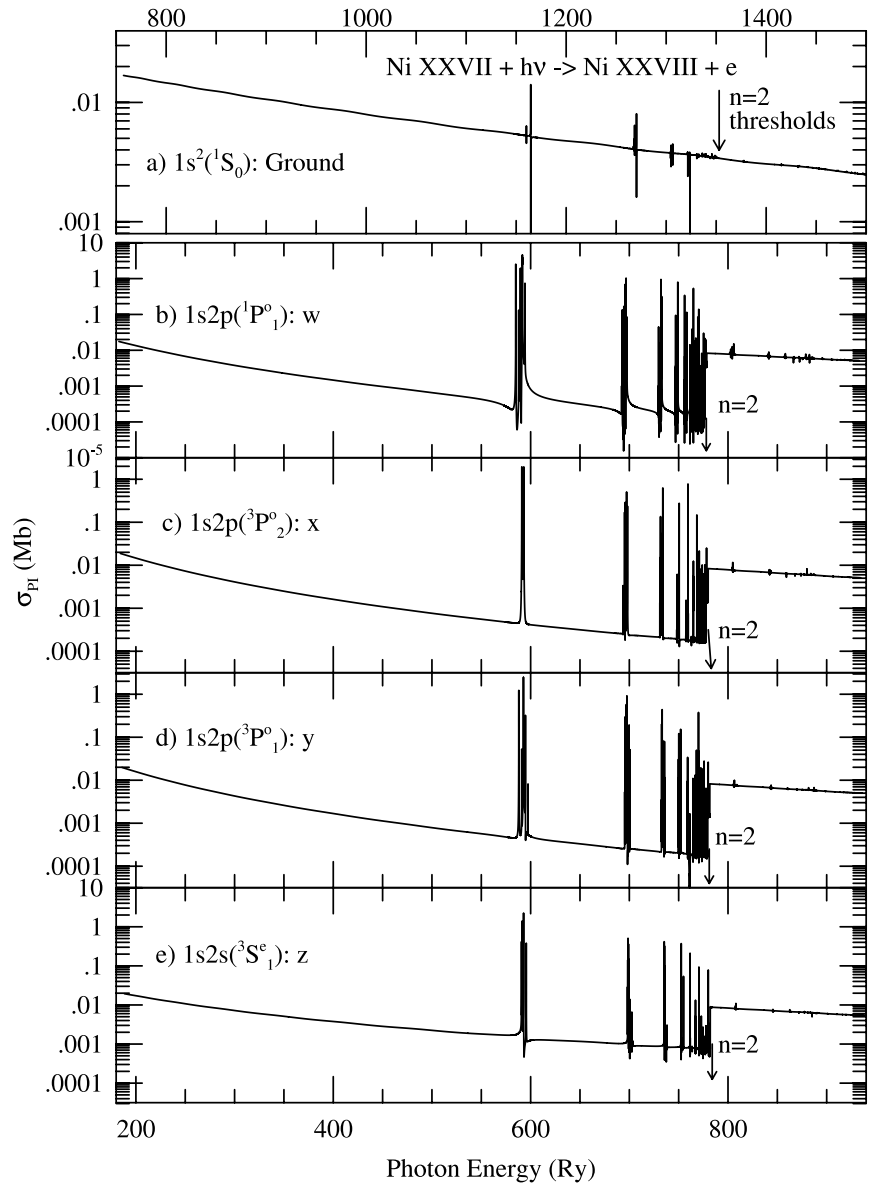

FIG. 6.-Level-specific photoionization cross sections of $(a)$ the ground $1 s^{2}\left({ }^{1} S_{0}\right)$ and excited $(b) 1 s 2 p\left({ }^{1} P_{1}^{o}\right)$-w, (c) $1 s 2 p\left({ }^{3} P_{2}^{o}\right)$-x, $(d) 1 s 2 p\left({ }^{3} P_{1}^{o}\right)$-y, and (e) $1 s 2 s\left({ }^{3} S_{1}\right)$-z levels of Ni xxvII. The excited levels correspond to the prominent $\mathrm{X}$-ray lines: resonance $(\mathrm{w})$, intercombination $(\mathrm{y})$, and forbidden $(\mathrm{x}, \mathrm{z})$ and show K-shell ionization edge at $n=2$ thresholds.

are KLL, KLM, KLN, etc., going up to the $n=2$ threshold and KMM, KMN, etc., going up to the $n=3$ threshold, where KLL means $1 s 2 l 2 l$, KLM means $1 s 2 l 3 l^{\prime}$, etc. These resonances manifest themselves as dielectronic satellite lines observed in tokamaks, electron-beam-ion-traps (EBIT), ion storage rings, and astrophysical sources. The KLL complexes have been well studied in previous works (e.g., Gabriel 1972; Pradhan \& Zhang 1997; Zhang et al. 1999; Oelgoetz \& Pradhan 2001), for various ions.

We express the photorecombination rates in terms of photoelectron energy,

$$
\alpha_{\mathrm{RC}}(E)=v \sigma_{\mathrm{RC}}(E),
$$

where $v$ is the photoelectron energy, an experimentally measurable quantity. Figure $3 b$ presents the expanded part of the resonances up to $n=2$ threshold in $\alpha_{\mathrm{RC}}(E)$ of Ni xxvI. The observed shape corresponds to the detailed $\alpha_{\mathrm{RC}}(E)$ convolved by the monochromatic bandwidth of the experiment (e.g., for C III by Pradhan et al. 2001).

Level-specific recombination rate coefficients $\alpha_{\mathrm{RC}}(T)$ of 98 levels are presented for Ni XxvI. They correspond to all associated $J \pi$ levels $i \equiv n(S L J)$ with $n \leq 10$ and $\ell \leq 9$. Figure 4 presents $\alpha_{i}(T)$ into the eight lowest excited $n=2$ and 3 levels of $n(S L J): 2 s^{2} S_{0}, 2 p{ }^{2} P_{1 / 2,3 / 2}^{o}, 3 s{ }^{2} S_{0}, 3 p{ }^{2} P_{1 / 2,3 / 2}^{o}$, and 


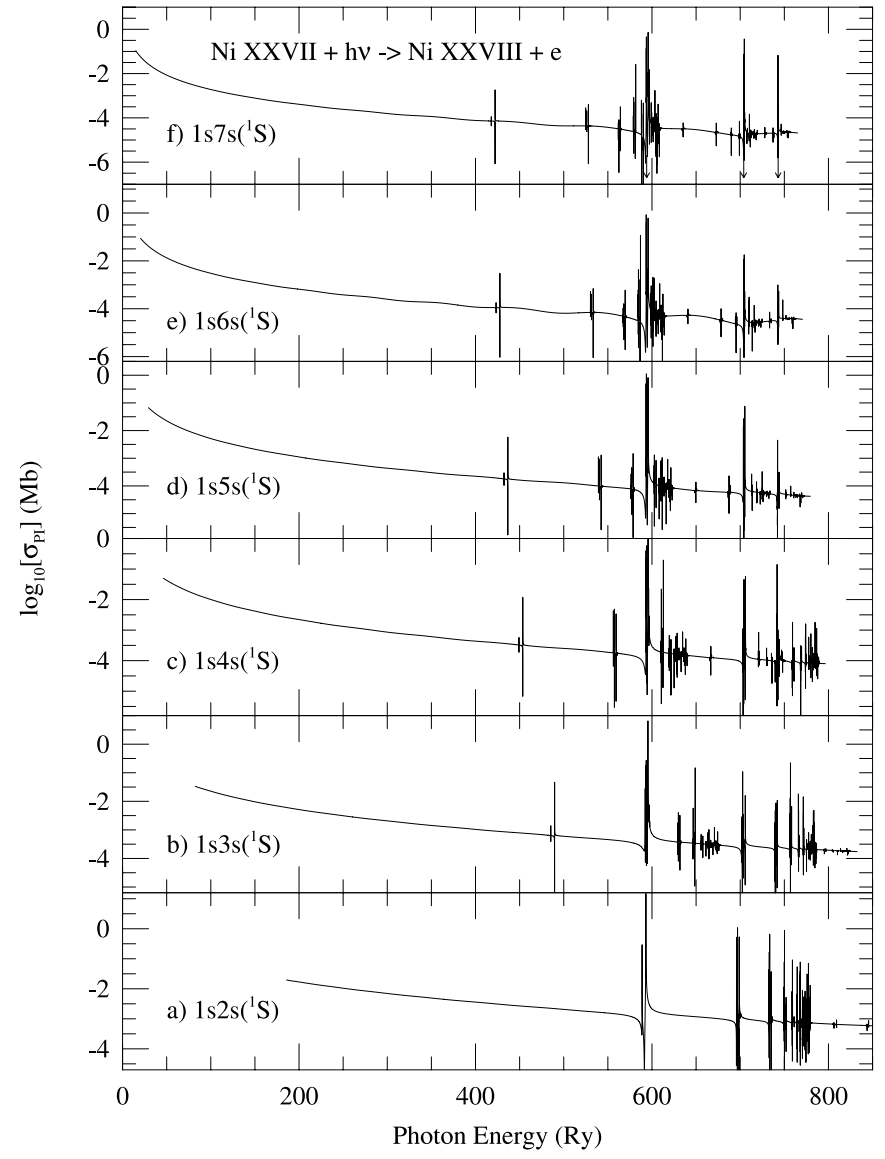

FIG. 7.-Partial photoionization cross sections of the Rydberg series of levels 1sns $\left({ }^{1} S_{0}\right), 2 \leq n \leq 7$, of Ni XXVII into the ground level $1 s\left({ }^{2} S_{1 / 2}\right)$ of the core, displaying PEC (photoexcitation-of-core) resonances at about 594, 704, and 743 ryd (arrows in top panel) of core levels ${ }^{2} P_{1 / 2,3 / 2}^{o}$ of configurations $2 p, 3 P$, and $4 p$.

$3 d^{2} D_{3 / 2,5 / 2}$. These rates are relatively smooth except for a small and diffuse DR "bump" at high temperatures.

Total recombination rates are given in Table 3 . The main features are illustrated and compared with previously available data for RR and DR rates in Figure 5. The solid curve in the figure is the BPRM total unified $\alpha_{\mathrm{RC}}(T)$ and shows typical features. We start with a higher rate coefficient at very low temperatures, because of the dominance of RR into an infinity of high- $n$ levels. The value of $\alpha_{\mathrm{RC}}(T)$ decreases with increasing $T$ until high temperatures, where it rises because of the dominance of DR, followed by a monotonic decay.

The present total unified recombination rate coefficients, $\alpha_{\mathrm{RC}}(T)$, for Ni XXVI are compared with RR rate coefficients (dashed line) by Verner \& Ferland (1996), DR rates (dotted line) by Jacobs et al. (1980), and DR rates (chain-dashed line) by Romanik (1988). The present rates agree very well with the previously calculated rates, especially with the sum of RR + DR (dot-dashed line) of Verner \& Ferland and of Jacobs et al.

\subsection{Ni XXVII}

A total of 198 bound levels are found for $\mathrm{Ni}$ XXVII with $n \leq 10,0 \leq l \leq 9,0 \leq L \leq 14$, and total angular momentum of $0 \leq J \leq 10$.

\subsubsection{Photoionization}

Partial and total photoionization cross sections are presented for all 198 bound levels $(n \leq 10)$ of Ni XxVII. Illustrative results are presented in Figures 6 and 7.

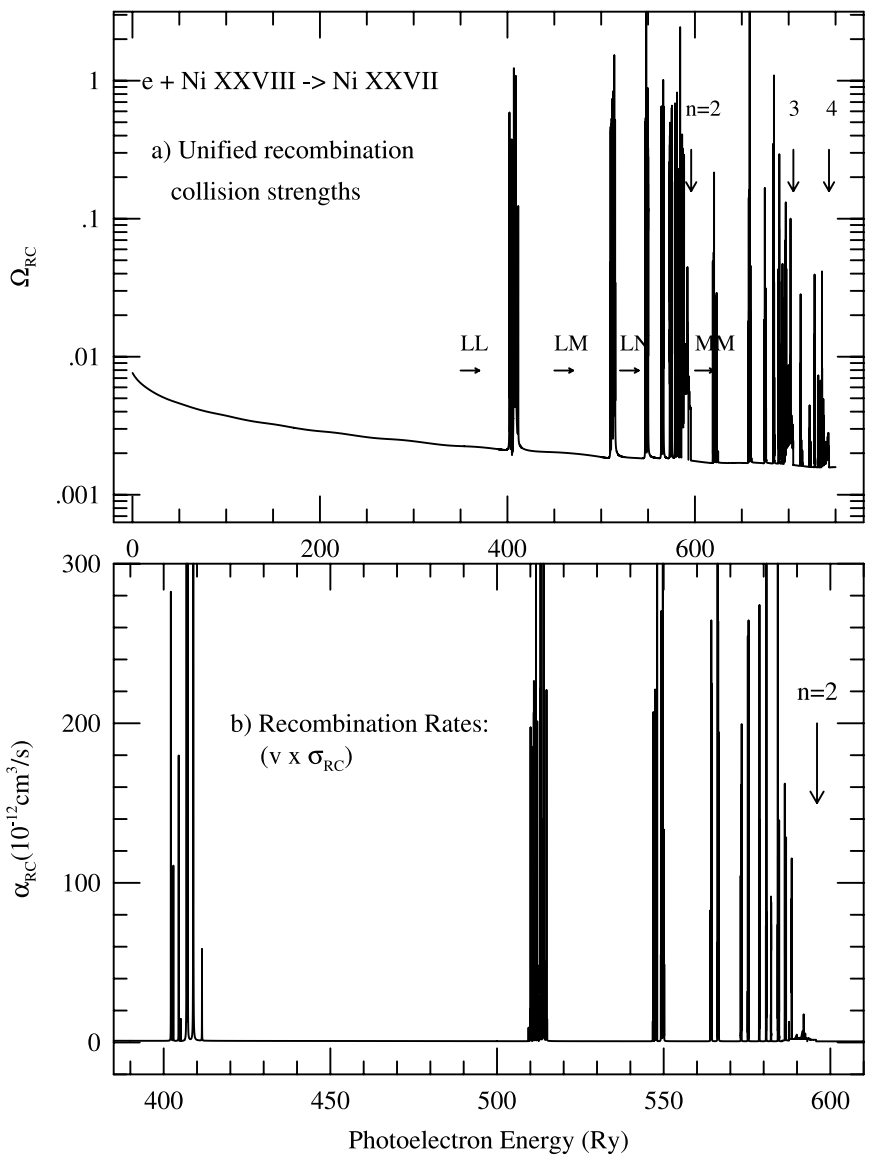

FIG. 8.- (a) Total unified $\left(e+\right.$ ion) recombination collision strengths, $\Omega_{\mathrm{RC}}$ and $(b)$ unified recombination rate coefficients, $\alpha_{\mathrm{RC}}(E)$ with photoelectron energy of Ni xxVII. Note the separated resonance complexes, LL, LM, etc., of $n=2$ and MM, MN, etc., of $n=3$ and NN, NO, etc., of $n=4$ thresholds. The quantity $\alpha_{\mathrm{RC}}(E)$, convolved with a bandwidth, is measurable.

Figure 6 presents samples of level-specific photoionization cross sections of Ni XxviI. The ground $1 s^{2}\left({ }^{1} S_{0}\right)$ level cross sections are in the topmost panel, while the lower four panels present $\sigma_{\mathrm{PI}}$ of the four lowest $n=2$ excited levels of Ni XXVII. These excited levels correspond to the prominent lines of the $\mathrm{K} \alpha$ complex in the X-ray emission of He-like ions: resonance [w: $\left.1 s^{2}\left({ }^{1} S_{0}\right) \leftarrow 1 s 2 p\left({ }^{1} P_{1}^{o}\right)\right]$, intercombination $\left[\mathrm{y}: 1 s^{2}\left({ }^{1} S_{0}\right) \leftarrow\right.$ $\left.1 s 2 p\left({ }^{3} P_{1}^{o}\right)\right]$, and forbidden $\left[\mathrm{x}: 1 s^{2}\left({ }^{1} S_{0}\right) \leftarrow 1 s 2 p\left({ }^{3} P_{2}^{o}\right)\right.$ and $\mathrm{z}$ : $\left.1 s^{2}\left({ }^{1} S_{0}\right) \leftarrow 1 s 2 s\left({ }^{3} S_{1}\right)\right]$ lines, respectively, and yield valuable spectral diagnostics of temperature, density, ionization balance, and abundances in the plasma source.

In the photoionization cross section of the ground level $\left(1 s^{2}{ }^{1} S_{0}\right)$ of Ni XxvII, the Rydberg series of resonances, KL and $\mathrm{K} n(n>2)$, begin at fairly high energies owing to the high $n=2$ excitation thresholds of Ni XxVIII. However, the ground level $\sigma_{\mathrm{PI}}$ of Ni XXVII does not show a significant K-shell jump at $n=2$ threshold, as seen in Ni Xxvi. Nonetheless, the K-shell ionization jump at the $n=2$ target levels

$$
h \nu+\mathrm{Ni} \operatorname{XXVII}(1 s 2 s, 1 s 2 p) \rightarrow e+\mathrm{Ni} \operatorname{XXVIII}(2 s, 2 p)
$$

can be seen clearly in the photoionization cross sections in Figures $6 b-6 e$.

Figure 7 presents partial photoionization cross sections of the Rydberg series of $J=0$ levels, $1 \operatorname{sns}\left({ }^{1} S_{0}\right)$, where $2 \leq n \leq 7$, of Ni XXVII ionizing into the ground level $1 s\left({ }^{2} S_{1 / 2}\right)$ of the core. The figure displays the PEC resonances at about 594, 704, and 


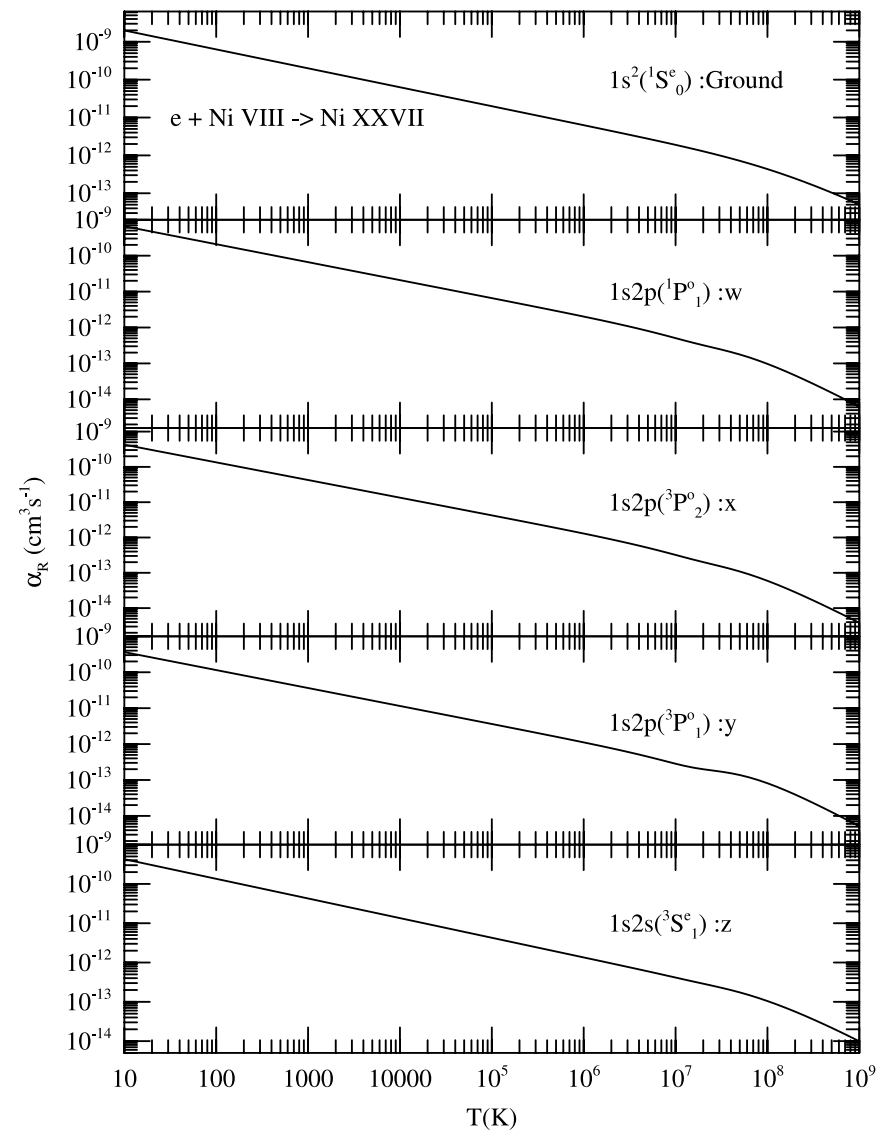

FIG. 9.-Level-specific recombination rate coefficients for Ni XXVII into the ground and excited $n=2$ levels responsible for the prominent X-ray $w, x, y$, and $z$ lines.

743 ryd (marked by arrows in the top panel) due to core transitions to allowed levels. The energy positions correspond to core levels ${ }^{2} P_{1 / 2}^{o},{ }^{2} P_{3 / 2}^{o}$ of configurations $2 p, 3 P$, and $4 p$.

\subsubsection{Electron-Ion Recombination}

The total unified photorecombination collision strength $\Omega_{\mathrm{RC}}$ for $\mathrm{Ni}$ XXVII is presented in Figure $8 a$. It corresponds to summed contributions from photoionization cross sections of all levels and high- $n$ DR. The strength $\Omega_{\mathrm{RC}}$ decays smoothly with energy until resonance complexes appear at very high energy, as expected for a He-like ion. The resonance complexes are LL, LM, $\mathrm{LN}$, etc., going up to the $n=2$ threshold, MM, MN, etc., going up to the $n=3$ threshold, and NN, NO, etc., going up to the $n=4$ threshold; LL means $2 l 2 l$, LM means $2 l 3 l^{\prime}$, etc. The $n=4$ resonances are too weak for any significant contributions.

Figure $8 b$ presents an expanded part of the resonances up to the $n=2$ threshold of photorecombination rates $\left[\alpha_{\mathrm{RC}}(E)\right]$ varying with photoelectron energy. As mentioned before, $\alpha_{\mathrm{RC}}(E)$ is a measurable quantity, and the observed shape is usually convolved by the monochromatic bandwidth of the experiment.

Level-specific recombination rate coefficients are obtained for 198 levels of $n(S L J)$ with $0 \leq J \leq 10$ and $n \leq 10$. Figure 9 presents level-specific rates for the ground and the $n=2$ levels corresponding to the X-ray $w, x, y$, and $z$ lines of $\mathrm{Ni}$ XXVII. The rates show a relatively smooth decrease with temperature.

The total unified recombination rate coefficients are given in Table 3. The main features are illustrated and compared with previously available data for RR and DR rates in Figure 10. The BPRM total unified $\alpha_{\mathrm{RC}}(T)$ (solid curve) shows typical features.

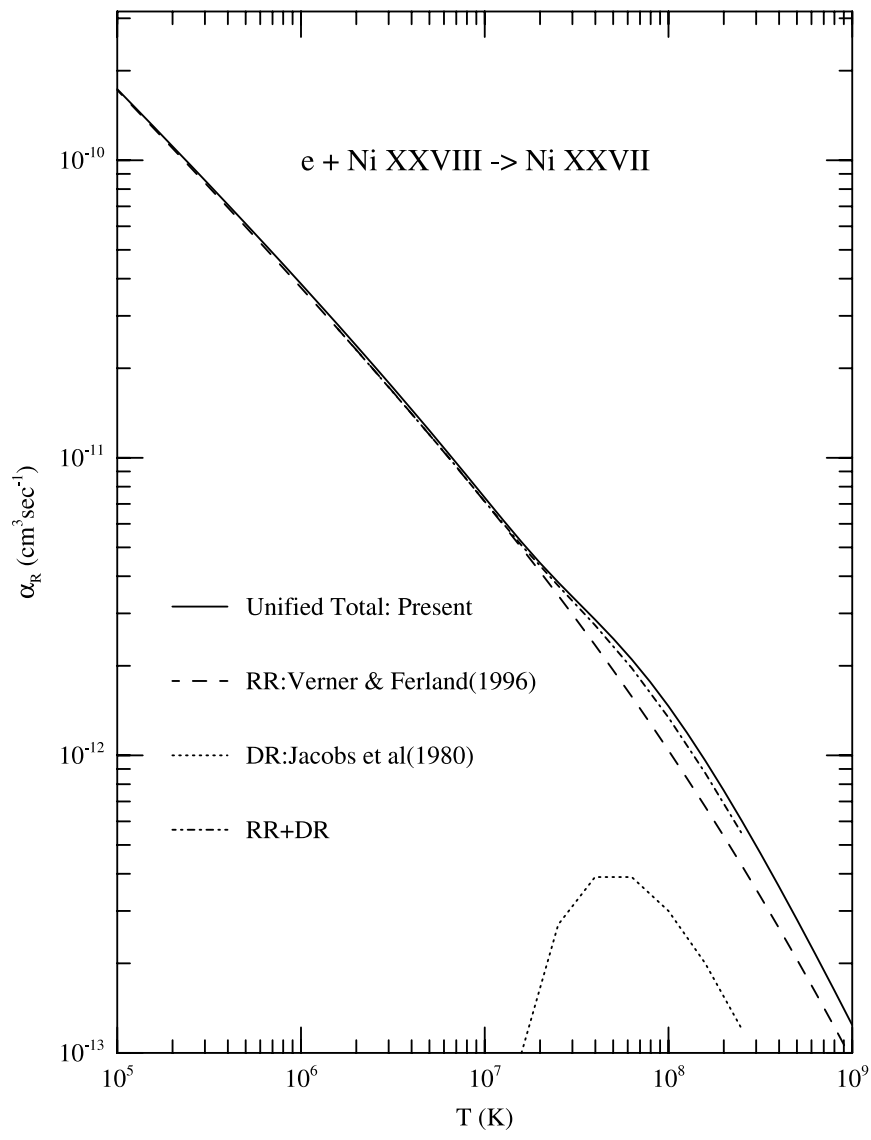

FIG. 10.-Total unified recombination rate coefficients, $\alpha_{\mathrm{RC}}(T)$, for Ni XXVII: present unified total (solid line), RR rates (dashed line) by Verner \& Ferland (1996), and DR rates (dotted line) by Jacobs et al. (1980).

The high recombination rate coefficients at very low temperatures, owing to the dominance of RR into an infinity of high- $n$ levels. decreases with increasing $T$ until high temperatures, where it shows a "shoulder" because of the dominance of DR, which is followed by a monotonic decay.

The present total unified recombination rate coefficients, $\alpha_{\mathrm{RC}}(T)$, for Ni XXVI are compared with RR rate coefficients (dashed line) by Verner \& Ferland (1996) and DR rates (dotted line) by Jacobs et al. (1980). The present rates agree very well with the previously calculated rates, especially with the sum of $\mathrm{RR}+\mathrm{DR}$ (dot-dashed line) of Verner \& Ferland and of Jacobs et al.

\section{CONCLUSION}

Extensive results from relativistic calculations for total and level-specific photoionization and recombination cross sections and rates are presented for Ni XXVI and Ni XXVII. These are of general interest in UV and X-ray spectroscopy of laboratory and astrophysical sources.

The present level-specific data can be used to construct recombination-cascade matrices for $\mathrm{Ni}$ XXVI and $\mathrm{Ni}$ XXVII to obtain effective recombination rates into specific fine-structure levels $n(S L J)$ with $n \leq 10$ and $\ell \leq n-1$ (e.g., Pradhan 1985). The present total unified recombination rates agree very well with the sum of RR and DR of previous calculations. It is expected for the He- and Li-like ions for which electron-electron correlation is weak, resulting in a small interference between RR and DR. However, the unified method for recombination provides level-specific rates of hundreds of levels and corresponding 
self-consistent photoionization cross sections of many bound levels, not obtainable by other existing methods. The present data are more than sufficient for extrapolation to high- $n, \ell$ necessary to account for all cascade contributions.

The available data include (i) photoionization cross sections, both total and partial, for bound fine-structure levels of $\mathrm{Ni}$ XXVI and Ni XxvII up to the $n=10$ levels; (ii) total unified recombination rate coefficients for $\mathrm{Ni}$ XXVI and Ni XXVII, and levelspecific recombination rate coefficients for levels up to $n=10$; and (iii) total unified recombination collision strength, cross sections, and rates with photoelectron energies of Ni XxvI and Ni XxVII. Further calculations for other He-like and Li-like ions are in progress. All photoionization and recombination data are available electronically from the author.

This work was supported partially by NSF and NASA. The computational work was carried out on the Cray SV1 at the Ohio Supercomputer Center in Columbus, Ohio.
Bell, R. H., \& Seaton, M. J. 1985, J. Phys. B, 18, 1589

\section{REFERENCES}

Berrington, K. A., Burke, P. G., Butler, K., Seaton, M. J., Storey, P. J., Taylor, K. T., \& Yan, Y. 1987, J. Phys. B, 20, 6379

Berrington, K. A., Eissner, W., \& Norrington, P. H. 1995, Comput. Phys. Commun., 92, 290

Canizares, C., et al. 2000, in Atomic Data Needs in X-Ray Astronomy, ed. M. A Bautista, T. R. Kallman, \& A. K. Pradhan (NASA/CP-2000-209968), 5, http:// heasarc.gsfc.nasa.gov/docs/heasarc/atomic/proceed.html

Eissner, W., Jones, M., \& Nussbaumer, H. 1974, Comput. Phys. Commun., 8, 270

Gabriel, A. H. 1972, MNRAS, 160, 99

Hummer, D. G., Berrington, K. A., Eissner, W., Pradhan, A. K., Saraph, H. E., \& Tully, J. A. 1993, A\&A, 279, 298

Jacobs, V. L., Davis, J., Rogerson, J. E., Blaha, M., Cain, J., \& Davis, M. 1980, ApJ, 239, 1119

Nahar, S. N. 1996, Phys. Rev. A, 53, 2417

2002, A\&A, 389, 716

Nahar, S. N., \& Pradhan, A. K. 1992, Phys. Rev. Lett., 68, 1488
Nahar, S. N., \& Pradhan, A. K. 1994, Phys. Rev. A, 49, 1816 1997, ApJS, 111, 339

2000, Phys. Scr., 61, 675

2003, ApJS, 149, 239

Nahar, S. N., Pradhan, A. K., \& Zhang, H. L. 2000, ApJS, 131, 375 2001, ApJS, 133, 255

Oelgoetz, J., \& Pradhan, A. K. 2001, MNRAS, 327, L42

Pradhan, A. K. 1985, ApJ, 288, 824

Pradhan, A. K., Chen, G.-X., Nahar, S. N., \& Zhang, H. L. 2001, Phys. Rev. Lett., 87, 183201

Pradhan, A. K., \& Zhang, H. L. 1997, J. Phys. B, 30, L571

Romanik, C. 1988, ApJ, 330, 1022

Sakimoto, K., Terao, M., \& Berrington, K. A. 1990, Phys. Rev. A, 42, 291

Scott, N. S., \& Burke, P. G. 1980, J. Phys. B, 13, 4299

Scott, N. S., \& Taylor, K. T. 1982, Comput. Phys. Commun., 25, 347

Seaton, M. J. 1987, J. Phys. B, 20, 6363

Verner, D. A., \& Ferland, G. 1996, ApJS, 103, 467

Zhang, H. L., Nahar, S. N., \& Pradhan, A. K. 1999, J. Phys. B, 32, 1459 